\title{
Original
}

\section{Assessment of the Daily Variation in Urinary Symptoms in Patients with Benign Prostatic Hyperplasia Using 24-Hr Home Uroflowmetry}

\author{
Naotaka Ishidori, Makoto SHIMAdA and Hideki YoshidA
}

\begin{abstract}
We used home uroflowmetry to assess urinary excretion over a 24-hr period and used the results of this study to evaluate the reliability of the International Prostate Symptom Score (IPSS) and to determine the daily variation in urinary excretion. Fifteen patients (mean age 68.3 years) with benign prostatic hyperplasia (BPH) were examined using home uroflowmetry. These patients also completed the IPSS questionnaire. The IPSS score was checked for agreement with the data recorded by home uroflowmetry. Urinations were classified into the following 4 periods. The 24-hr period was divided into the active period and the sleeping period. The home uroflowmetry parameters were compared to these periods. The IPSS scores for frequency and intermittency of urination correlated well with the respective home uroflowmetry scores. Regarding daily variation, maximum flow rate (Qmax) was often highest in the evening and lowest at night, and the voided volume and voiding time tended to be highest at night, and lowest in the afternoon. The mean Qmax and mean average flow rate (Qave) were significantly higher during the active period than during the sleeping period. In contrast, the mean voided volume and mean voiding time were significantly higher values during the sleeping period than during the active period. Two items on the IPSS questionnaire, frequency and interruption of the urinary stream, were found to be highly reliable. Qmax and Qave values varied during the 24-hr period and were an estimated $10 \%$ and $20 \%$ higher, respectively, during the active period than during sleeping period.
\end{abstract}

Key words : IPSS (International Prostate Symptom Score), home uroflowmetry, BPH (benign prostatic hyperplasia)

\section{Introduction}

The International Prostate Symptom Score (IPSS) has been proposed for assessing the severity of benign prostatic hyperplasia (BPH), choosing therapy, and evaluating the effectiveness of treatment ${ }^{1}$. This score is based on the AUA Symptom Score established by the American Urological Association (AUA) to numerically express the severity of urinary symptoms, and has been supported by international conferences on BPH. For this reason, the IPSS is the most commonly used score to assess prostate symptoms. Its reliability and

Department of Urology, Showa University School of Medicine, 1-5-8 Hatanodai, Sinagawa-ku, Tokyo 142-8666, Japan. 
characteristics have been widely discussed and it has been reported to be useful in evaluating the effectiveness of treatment for $\mathbf{B P H}^{2}$. However, it has also been reported that there is only a weak correlation between the symptom score, the degree of prostatic enlargement, and parameters of lower urinary tract obstruction ${ }^{3)}$. Also, the reproducibility of the IPSS has been questioned ${ }^{4)}$ and it has been reported that it should not be used for making a diagnosis of $\mathbf{B P H}^{5)}$. Actual use of the IPSS makes it difficult to answer the questionnaire in such frequency as less than 1 time in 5 or about half the time.

Many patients show differences in urinary symptoms between day and night, and some reports have indicated that Uroflowmetry produces widely varying results at different times during a 24-hr period ${ }^{6)}$. However, daily variation of urinary excretion has not yet been confirmed.

In this study, we used home uroflowmetry to assess urinary excretion over a 24-hr period at home and evaluated (1) the reliability of the IPSS and (2) the daily variation in urinary excretion, in order to make a precise assessment of urinary symptoms in patients with BPH.

\section{Materials and Methods}

The patients included in this study were males aged 60-77 years (mean age : 68.3 years) who had BPH diagnosed by transrectal ultrasonography or digital rectal examination. In addition to patients with systemic complications, such as diabetes mellitus and heart disease, we excluded those with a neurogenic bladder, bladder neck obstruction, urethral stricture, and prostate cancer, which were diagnosed by intravenous urography, urethrocystography, cystometry, and biopsy of the prostate, respectively. On visiting an outpatient clinic office, the subjects were provided with a home uroflowmeter (Dantec, the Da Capo ${ }^{\mathrm{TM}}$ Home Uroflowmeter) to monitor all urinations. After the device was returned, the data was entered into a personal computer. All subjects were required to report the time of awakening and retiring to bed, and this information was entered into the home uroflowmeter. Thirteen subjects also completed the IPSS questionnaire before home monitoring.

The scores obtained from the IPSS questionnaire for the frequency of urination, intermittency of urination, and frequency of nocturia, were compared to the data recorded by the home uroflowmeter. For comparison with IPSS scores, the respective incidence of the above 3 items recorded by the home uroflowmeter was divided by the total number of urinations recorded, and the values (\%) were designated as follows: $0 \%=$ not at all, $1-$ $19 \%=$ less than 1 time in 5, 20-39\%=less than half the time, $40-59 \%=$ about half the time, $60-79 \%=$ more than half the time, and $80 \%=$ almost always. Urination recorded within 2 hours of the previous urination, during the day or at night, was defined as frequent urination. A polymodal urinary flow curve was defined as interruption of the urinary stream, and likewise when it was difficult to judge whether dribbling had occurred this was also defined as interruption of the urinary stream. The time of awakening and retiring to bed were recorded before commencing the 24-hr period of home uroflowmetry. All urinations occurring between bedtime and awakening were defined as frequency of nocturia. All urinations recorded by the home uroflowmeter were classified into the following 4 periods: (1) the morning period (from awakening to $12: 00$ ) ; (2) the afternoon period (from 12:00 to $18: 00$ ); (3) the evening period (from 18:00 to bedtime); and (4) the nocturnal period (from bedtime to awakening). 
Table 1. Correlation of results from the IPSS questionnaire and home uroflowmetry

\begin{tabular}{lcc}
\hline \multicolumn{1}{c}{ item of IPSS } & correlation coefficient $(\mathbf{r})$ & $\mathrm{p}$ value \\
\hline frequency of urination & 0.606 & 0.028 \\
intermittency of urination & 0.565 & 0.044 \\
frequency of nocturia & -0.246 & 0.396 \\
\hline
\end{tabular}

Table 2. Timing of the maximum and minimum values for Qmax, Qave, voided volume and voiding time

\begin{tabular}{llcccc}
\hline \multicolumn{2}{c}{ uroflow parameter } & morning & afternoon & evening & nocturnal \\
\hline \multirow{2}{*}{ Qmax } & maximum & 13 & 33 & $\mathbf{4 1}$ & 13 \\
& minimum & 20 & 20 & 27 & 33 \\
Qave & maximum & 0 & $\mathbf{6 6}$ & 27 & 7 \\
\multirow{4}{*}{ voided volume } & minimum & $\mathbf{4 0}$ & 7 & 20 & 33 \\
\multirow{3}{*}{ voiding time } & maximum & 33 & 13 & 13 & $\mathbf{4 1}$ \\
& minimum & 20 & $\mathbf{4 0}$ & 33 & 7 \\
& maximum & 40 & 0 & 13 & $\mathbf{4 7}$ \\
& minimum & 13 & $\mathbf{5 4}$ & 33 & 0 \\
\hline
\end{tabular}

morning period : from awakening to $12: 00$, afternoon period : from 12:00 to $18: 00$ evening period : from 18:00 to bedtime, nocturnal period : from bedtime to awakening

The maximum flow rate (Qmax), the average flow rate (Qave), the voided volume, and the voiding time of the 15 patients were averaged and the timing of the maximum and minimum values for these parameters was determined. Periods (1) to (3) were defined as the active period (from awakening to bedtime) and period (4) was defined as the sleeping period. The intra-day variation of urination was then averaged for all patients and mean values were calculated for Qmax, Qave, mean voided volume, and mean voiding time. Wilcoxon's rank sum test was performed, which corresponded to Spearman's rank correlation coefficient.

\section{Results}

The average prostate volume was $32.5 \mathrm{~g}$ (range : 20.9-54 g), and the average IPSS score was 14.8 (range: 6-23). The average number of urinations recorded for each patient by the home uroflowmeter was 20.3 and the average number of days of recording was 3.6. The total number of urinations for all 15 patients was 308 , of which $294(95.5 \%)$ were correctly measured.

The IPSS scores for frequent urination and interruption of the urinary stream determined for 13 patients gave correlation coefficients of 0.606 and 0.565 , respectively, when compared to the corresponding scores obtained by the home uroflowmeter. These correlations were significant $(p=0.028$ and 0.044$)$. However, the IPSS score for nocturia showed a correlation coefficient of only -0.246 for the corresponding home uroflowmetry score, this 
Table 3. Comparison between the active period and sleeping period of the uroflow parameters used by home uroflowmetry

\begin{tabular}{|c|c|c|c|}
\hline uroflow parameter & & $\mathrm{n}$ & mean $\pm \mathrm{SD}$ \\
\hline \multirow[t]{3}{*}{ Mean Qmzx (ml/s) } & & 15 & \\
\hline & Active period & & $10.4 \pm 4.2$ \\
\hline & Sleeping period & & $9.4 \pm 3.9 *$ \\
\hline \multirow[t]{3}{*}{ Mean Qave ( $\mathrm{ml} / \mathrm{s})$} & & 15 & \\
\hline & Active period & & $6.2 \pm 2.2$ \\
\hline & Sleeping period & & $5.2 \pm 2.4 *$ \\
\hline \multirow[t]{3}{*}{ Mean voided volume $(\mathrm{ml})$} & & 15 & \\
\hline & Active period & & $153.9 \pm 69.3$ \\
\hline & Sleeping period & & $224.7 \pm 191.3 *$ \\
\hline \multirow[t]{3}{*}{ Mean voiding time $(s)$} & & 15 & \\
\hline & Active period & & $31.0 \pm 14.7$ \\
\hline & Sleeping period & & $45.5 \pm 18.6 *$ \\
\hline
\end{tabular}

correlation was not significant $(\mathrm{p}=0.396)$ (Table 1$)$.

Regarding daily variation, Qmax was highest in the evening in 6 patients $(41 \%)$ and lowest at night in 5 patients $(38 \%)$. The voided volume and voiding time were highest at night in $6(41 \%)$ and 7 cases $(45 \%)$, respectively, however these parameters were lowest in the afternoon in $6(40 \%)$ and 8 cases $(54 \%)$, respectively (Table 2). The mean Qmax and mean Qave were significantly higher (both $\mathrm{p}<0.05$ ) during the active period than during the sleeping period. In contrast, the mean voided volume and mean voiding time were significantly higher (both $\mathbf{p}<0.05$, respectively) during the sleeping period than during the active period (Table 3).

\section{Discussion}

The assessment of urination by uroflowmetry can be influenced by the psychological suppression of urination. Measurements are usually made in a laboratory or an outpatient clinic and in such environments the patient is often under stress. This results in some patients being unable to urinate and some complain that urinary conditions are different to normal. Home uroflowmetry is reported to provide more concrete and precise information than the data obtained from a single test with a laboratory uroflowmeter ${ }^{7)}$. For this reason, we used a home uroflowmeter to assess urinary symptoms in patients with BPH.

In a previous study, patients were subjected to a 24-hr uroflowmetric evaluation to assess whether scores from IPSS questionnaires agreed with data obtained by uroflowmetry ${ }^{8}$. The scores correlated highly for nocturia and interruption of the urinary stream, while the correlation with frequency was weaker. In this study we used home uroflowmetry to assess the IPSS questionnaire items for frequency, interruption of the urinary stream, and nocturia, and found that frequency and interruption of the stream reflected actual urination with a high degree of reliability. Home uroflowmetry allows the evaluation of urination under conditions as close as possible to normal daily life, rather than under conditions of restricted activity or hospitalization. Among 13 subjects, 7 showed a higher IPSS score for nocturia 
than was recorded by uroflowmetric monitoring of urination. This may be because the patients included urination on awakening with nocturia when answering the IPSS questionnaire. When home uroflowmetry was used to measure nocturia, the results varied depending on the day of measurement. Among 13 patients, 4 reported the most frequent urinations within a few days as measured by home uroflowmetry as an IPSS score. It is possible that these patients would complain strongly of these symptoms. It is necessary for patients to be fully educated on these matters at the time of receiving the home uroflowmeter and answering the IPSS questionnaire.

Boci et al. ${ }^{9)}$ and Jacob et al..$^{6}$ investigated the daily variation of urination and reported differences between day and night. We also found two marked differences. The mean Qmax and mean Qave were significantly higher during the active period, with values at an estimated $10 \%$ and $20 \%$ respectively, and the mean voided volume and mean voiding time were significantly higher during the sleeping period, both estimated at $50 \%$. These findings suggest that excess urine tends to accumulate during the night at a time when patients took longer to pass urine and their flow was weak. Whether an increase of urine output during the night and early morning has some relationship with nocturia remains unknown, and needs further investigated. Another possibility is that the desire for micturition may be less at the same bladder capacity during the sleeping period. The weak urinary flow is explained by the fact that Qmax and Qave depend on the voided volume and increase as the volume increases ${ }^{10)}$. However, residual urine volume is related to bladder capacity and is not measured by home uroflowmetry. So, this study was unable to assess the correlation between voided volume and Qmax and Qave during the night because residual urine volume could not be measured. Our results confirmed that the mean voided volume was $153.9 \mathrm{ml}$ during the active period and $224.7 \mathrm{ml}$ during the sleeping period, which were lower volumes than those reported in other studies. The residual urine volume, as described above, may explain these lower volumes. Alternatively, these volumes may be usual for urination at home in patients with BPH. Further study is needed to assess residual urine volumes in these patients.

Two items on the IPSS questionnaire, frequency and interruption of the urinary stream, were found to be highly reliable.

Qmax and Qave values varied during the 24-hr period and were an estimated $10 \%$ and $20 \%$ higher, respectively, during the active period than during the sleeping period.

\section{References}

1) Barry MJ, Fowler FJ, Oleary MP, Bruskewitz RC, Holtgrewe HL, Mebust WK, Cockett ATK and The measurement committee of the american urological association: The american urological association symptom index for benign prostatic hyperplasia. J Urol 148 : 1549-1557 (1992)

2) Okazima E, Ozono S, Ota M, Tanaka M, Tani M, Hirao Y and Okajima E: A study on international prostate symptom score in the assessment of therapeutic effects and severity of symptoms due to benign prostatic hyperplasia. Jpn J Urol 86 : 1466-1474 (1995) (in Japanese)

3) Ko DSC, Fenster HN, Chambers K, Sullivan LD, Jens $M$ and Goldenberg SL: The correlation of multichannel urodynamic pressure-flow studies and american urological association symptom index in the evaluation of benign prostatic hyperplasia. J Urol 154 : 396-398 (1995)

4) Barry MJ, Girman CJ, Oleary MP, Walker-corkery ES, Binkowitz BS, Cockett ATK, Guess HA and The benign prostatic hyperplasia treatment outcomes study group: Using repeated measures of symptom score, uroflowmetry and prostate specific antigen in the clinical management of prostate disease. J Urol 153 : 99 103 (1995) 
5) Yalla SV, Sullivan MP, Lecanwasam HS, Dubeau CE, Vickers MA and Cravalho EG : Correlation of American Urological Association symptom index with obstructive and nonobstructive prostatism. J Urol 153: 674-680 (1995)

6) Golomb J, Lindner A, and Siegel Y and Korczak D : Variability and circadian changes in home uroflowmetry in patients with benign prostatic hyperplasia compared to normal controls. J Urol 147 : 1044-1047 (1992)

7) De la Rosette JJMCH, Witjes WPJ, Debruyne FMJ, Kersten PL and Wijkstra H: Improved reliability of uroflowmetry investigations: results of a portable home-based uroflowmetry study. Br J Urol 78 : 385-390 (1996)

8) Kimura A, Kurimoto S, Saiko Y, Hosaka Y, Kitamura T, Kawabe K, Nakamura S and Hamada C: Evaluation of reliability of IPSS by 24-hour uroflowmetry. Jpn J Urol 86 : 1728-1734 (1995) (in Japanese)

9) Boci R, Fall M, and Walden M, et al: Home Uroflowmetry: Improved accuracy in outflow assessment. Neurourol Urody 18 : 25-32 (1999)

10) Siroky MB, Olsson CA, and Krane RJ: The flow rate nomogram: I. Development. J Urol 122:665-668 (1979)

[Received February 5, 2001 : Accepted February 13, 2001] 